\title{
A NEW TEMPERATURE AND X-RAY MAPPING SYSTEM FOR 700-MHZ 5-CELL SUPERCONDUCTING CAVITIES*
}

\author{
T. Tajima ${ }^{\dagger}$, R. C. Gentzlinger, J. E. Ledford, M. A. Madrid, D. I. Montoya, A. H. Shapiro \\ Los Alamos National Laboratory, Los Alamos, NM 87545, USA
}

\begin{abstract}
A new system to map temperature and X-ray radiation around the external surface of $700-\mathrm{MHz}$ 5-cell superconducting cavities has been developed. It consists of an aluminum cylinder that is equipped with six modules of sensors. Eighty-one carbon resistors (temperature sensors) and seventy-one PIN diodes (X-ray sensors) are attached. This cylinder surrounds the 5-cell cavity and rotates about the cavity axis in about 6 minutes. A new feature, compared to the ones developed in the past, is its brush-contact mechanism on the outer surface of the aluminum cylinder, which enables the sensor array to rotate continuously in the same direction during the test. Although the present mechanism allows only one direction of rotation, it does not seem to be difficult to modify for both directions if electrical connections work in this manner. This paper describes the details of the structure and associated mechanisms as well as future schedule and plans of operation.
\end{abstract}

\section{INTRODUCTION}

Temperature/X-ray mapping identifies locations of losses inside the cavity, which permits understand the loss mechanism. There have been two types of temperature mapping system in the past, one is the fixed-board type [1-4] and the other is the rotating-arm type [5-7]. The fixed-arm type consists of tens of sensor-mounted boards around the cavity. The rotating-arm type consists of one sensor-mounted board on a certain meridian and this board rotates in the azimuthal direction. Since there is no mechanically moving part, fixed-type mapping can be very fast, e.g., 756 sensors can be scanned in less than $0.14 \mathrm{sec}[1]$. On the other hand, the rotating-arm type system is suited for large cavities [6,7] or multi-cell structures [5] that require a large number of sensors to cover all the surfaces. Another advantage of the rotatingarm type system is high spatial resolution in the azimuthal direction and that this resolution can be changed. At LANL, we have developed a rotating-arm type system for the temperature mapping of the APT $700-\mathrm{MHz} 5$-cell $(\beta=0.64)$ cavities [8].

\section{MAPPING SYSTEM}

The mapping system consists of temperature and X-ray sensors, sensor module boards, a drum for electrical connections between sensors and outside brushes, brushes

\footnotetext{
* Work supported by the US Department of Energy

† email: tajima@lanl.gov
}

for electrical connections to the cables that go to the connectors on the cryostat lid and data taking/display. Figure 1 shows a schematic of the structure when assembled with a 5-cell cavity. Figure 2 shows a schematic of sensors installed onto the module boards and inside of the drum conductors.

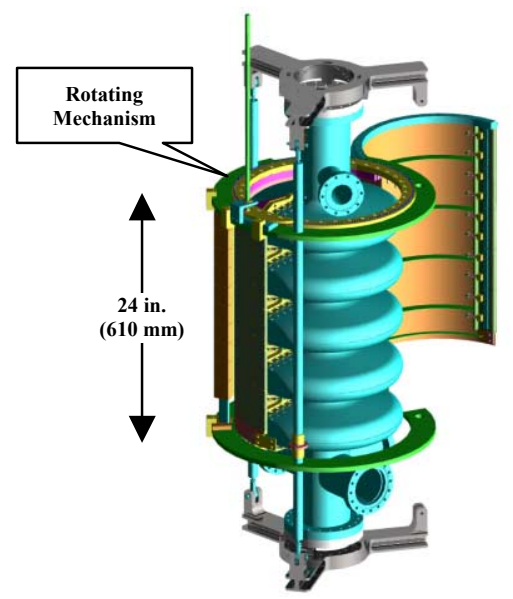

Figure 1: A conceptual drawing of the sensor board and the drum on which conductor panels are mounted.

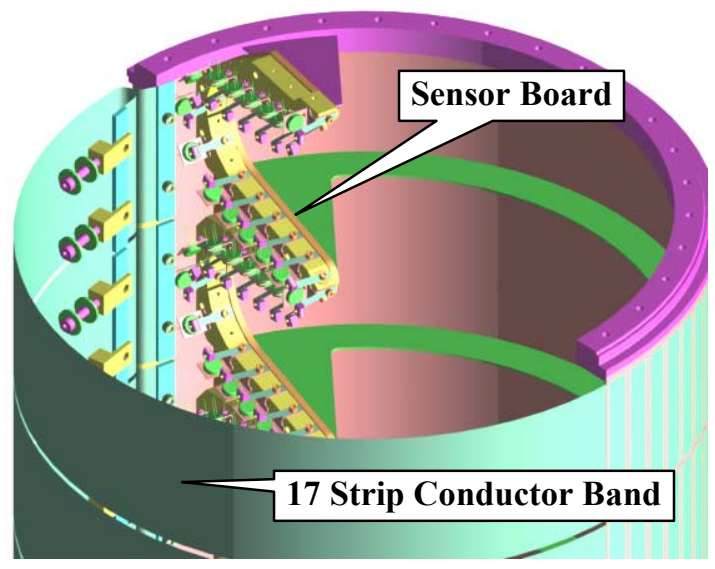

Figure 2: A drawing of the sensor boards attached inside the drum.

\subsection{Temperature and X-ray Sensors}

The temperature sensor is an Allen-Bradley $100 \Omega, 1 / 8$ $\mathrm{W}$ resistor embedded in a Vespel holder with Stycast. A test with 5 such sensors showed a sensitivity of $6.4-8.9$ $\Omega / \mathrm{mK}$ at $2-2.15 \mathrm{~K}$. The X-ray sensor is Hamamatsu 
S1722-02 PIN Silicon Photocell, which is the same as the one that has been used in KEK for their mapping system [6].

\subsection{Sensor module board}

Sensor boards consist of four middle boards as shown in Fig. 3, a top board and a bottom board. A middle board has 16 temperature sensors and $14 \mathrm{X}$-ray sensors. The top board has 9 temperature sensors and $8 \mathrm{X}$-ray sensors. The bottom board has 8 temperature sensors and 7 X-ray sensors. Thus, in total, there are 81 temperature sensors and $71 \mathrm{X}$-ray sensors.

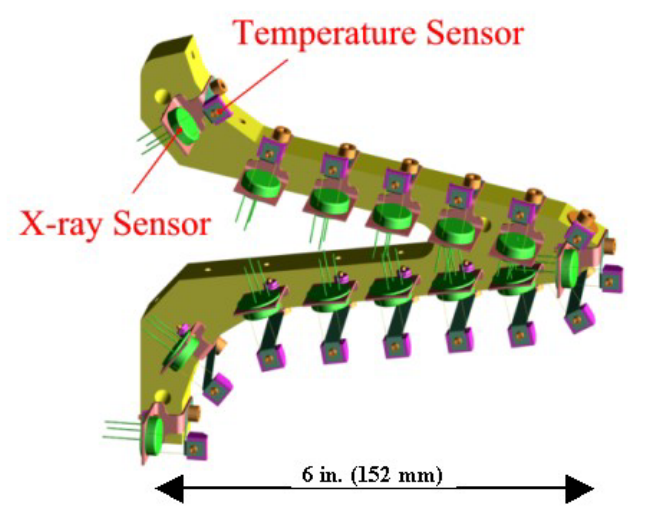

Figure 3: Temperature and X-ray sensor module.

\subsection{Rotating structure}

An 18-inch (457.2-mm) diameter drum made of two thin aluminum plates rotates around the cavity with the sensor boards. On the outside surface of the drum are five 0.005 -inch $(0.127-\mathrm{mm})$ thick Polyimide sheets on which a total of 1700.0014 -inch $(36-\mu \mathrm{m})$ thick copper strips are coated for electrical contacts. Each strip has a width of 0.09 inches $(2.286 \mathrm{~mm})$ and an interval of 0.12 inches $(3.048 \mathrm{~mm})$.

\subsection{Electrical contacts}

Electrical contacts between the conductor strips on the outer surface of the drum and the cables that exit the cryostat are made with 1700.006 -inch $(0.1524-\mathrm{mm})$ thick gold-plated phosphor-bronze brushes located outside of the drum and attached on a rod. To mitigate the thermal contraction during cool-down these brushes are divided into ten groups, each of which are mounted on an individual G-10 board and can be adjusted according to the positions of the Polyimide sheets. Figure 4 shows a drawing of one G-10 board with 17 brushes soldered onto copper strips. The width of the brush is 0.06 inches $(1.524 \mathrm{~mm})$ and the interval between each brush is 0.12 inches $(3.048 \mathrm{~mm})$.

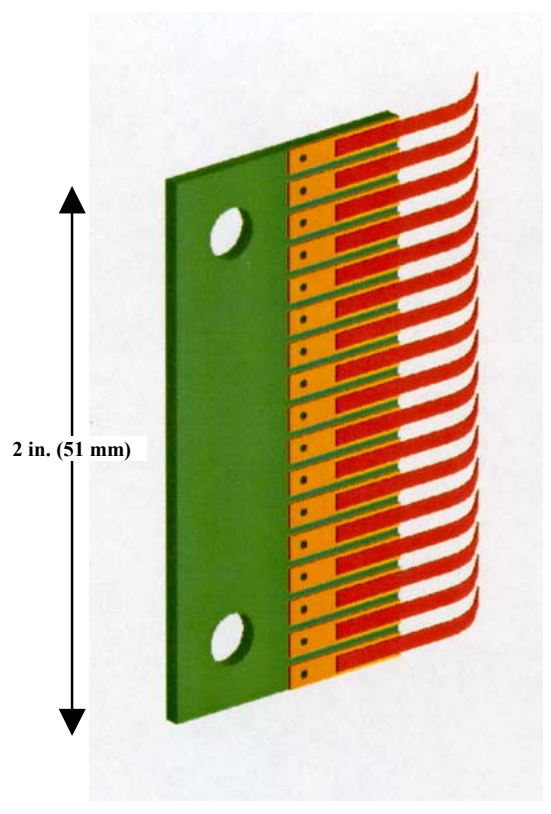

Figure 4: Brushes for electrical contacts on the outer surface of the drum.

\subsection{Rotation mechanism}

Figure 5 shows the rotating mechanism for the structure. The structure is rotated with a simple crankshaft lever. When the vertical rod at right rotates, the lever moves horizontally and picks up one of the 200 pins attached along the perimeter of a flange on top of the drum. There is a spring that assures holding of the pin (not shown in Fig. 5). Due to this mechanism, the rotation is only in one direction, clockwise as viewed from top.

To know the original position in case the data taking mal-functions, there is one longer pin and another pair of electrical contacts that gives a signal indicating one point along the circumference as shown at left in Fig. 5.

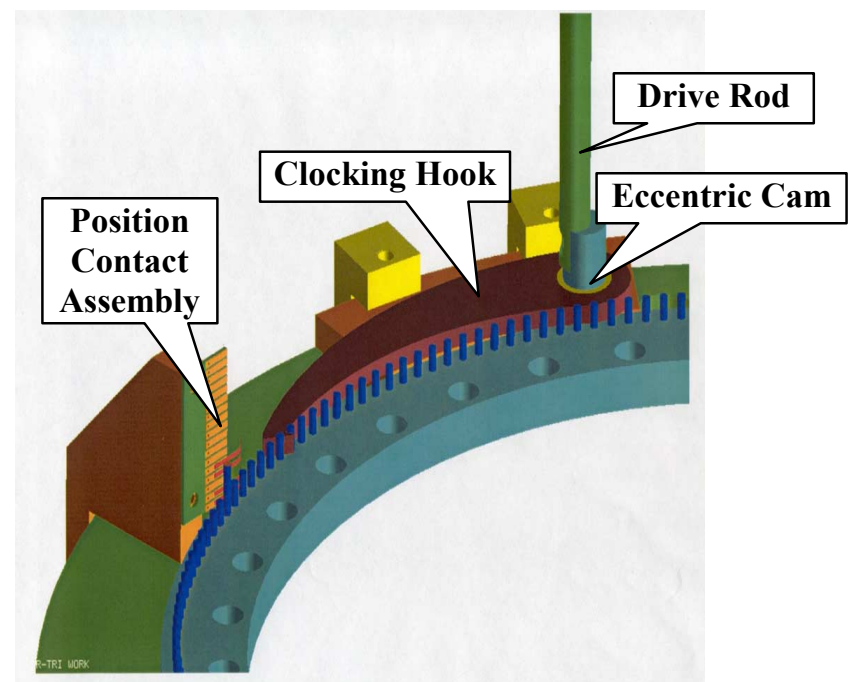

Figure 5: Rotation mechanism and sensing original point. 


\subsection{Data acquisition and display}

Though not yet attempted, our present plan is take 200 arrays of data that correspond to 200 meridians, i.e., 1.8 degrees between each meridian. We are planning to spend 2 seconds for one meridian, i.e., 1 second for the lever to move the drum from one pin to another and 1 second to take an array of data. Therefore, it will take 400 seconds to get a mapping of the entire surface.

Figure 6 shows an example of the display we are planning. Either 2-D or 3-D displays like Fig. 6 will be possible.

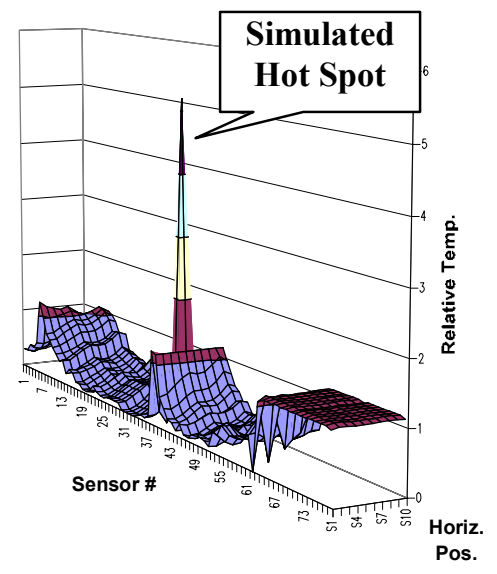

Figure 6: A simulation of a mapping display.

\section{SCHEDULE AND PLAN}

\subsection{Schedule}

Manufacturing of all the parts for the system is complete. We are planning to test the mechanism at room temperature in June 2001. Then, the system will be ready for the testing of 700-MHz 5-cell cavities in July 2001.

\subsection{Plan of Operation}

We are planning to start rotating the drum when we detect $\mathrm{Q}$ degradation and continue rotating it until we reach a maximum gradient.

Also, when we find heating spots, we plan to stop rotating the arm and take data repeatedly at a fixed meridian to see the time evolution of the heating and Xray radiation.

\section{SUMMARY}

At LANL, we have developed a temperature and X-ray mapping system to explore and identify the cause of field emissions of $700-\mathrm{MHz}, 5$-cell, $\beta=0.64$ cavities. The mapper is a rotating-arm type, consisting of sensor modules (a total of 81 temperature sensors and $71 \mathrm{X}$-ray sensors), a rotating aluminum drum that has conductor strips on the outside surface for electrical connection and brushes on the outside of the drum for electrical contacts. The system is nearly complete and to be tested soon.

\section{ACKNOWLEDGMENTS}

The authors would like to thank H. Haagenstad and A. Rendon for helping machining and assembly of some parts of the mapping system. Also, we appreciate support and encouragement of D. Schrage and D. Chan. Finally, we would like to thank P. Kelley for proof-reading the paper.

\section{REFERENCES}

[1] J. Knobloch, Ph. D. thesis, CLNS THESIS 97-3 (1997) p. 63.

[2] M. Pekeler, "High gradients in superconducting RF cavities," Proc. $8^{\text {th }}$ Workshop on RF Superconductivity, Abano Terme, Italy, 6-10 October 1997, p. 822.

[3] J. Lesrel, et al., "Thermal Behavior of SRF Cavities at High Gradients," Proc. EPAC'98, Stockholm, June 1998, p. 1861.

[4] T. Tajima, et al., "Temperature Mapping System Developed at KEK for Field Emission Studies on Superconducting Cavities," HEACC'92, Hamburg, July 1992. KEK Preprint 92-91.

[5] Q. S. Shu, et al., "A Novel Rotating Temperature and Radiation Mapping System in Superfluid He and Its Successful Diagnostics," Advances in Cryogenics Engineering, 41, p. 895 (1996).

[6] T. Takahashi, et al, "Development of Superconducting Cavity for KEK B-Factory," KEK Preprint 93-102 (1993).

[7] H. Piel, "Diagnostic Methods of Superconducting Cavities and Identification of Phenomena," Proc. $1^{\text {st }}$ Workshop on RF Superconductivity, Karlsruhe, Germany, July 1980, p. 85 .

[8] T. Tajima, et al., "Developments of 700-MHz 5-Cell Superconducting Cavities of APT," this conference. 